Kaushik Roy ${ }^{1, \star}$, Ambikesh K. Srivastwa ${ }^{1}$ and Chandan K. Ghosh ${ }^{1}$

\title{
Anticoagulant, thrombolytic and antibacterial activities of Euphorbia acruensis latex-mediated bioengineered silver nanoparticles
}

https://doi.org/10.1515/gps-2019-0029

Received October 25, 2018; accepted March 26, 2019.

Abstract: In this report, we present a simple and unexplored procedure for green synthesis of silver nanoparticles featuring exudation of Euphorbia acruensis along with the study of its antibacterial and anticoagulant properties. Analytical techniques like ultraviolet visible spectroscopy (UV-Vis), X-ray diffraction (XRD) and high resolution transmission electron microscopy (HRTEM) were used to analyse the production, crystallinity and morphology of bio-reduced silver nanoparticles. The antibacterial study was performed by following standard disc diffusion method. Most importantly, the anticoagulant and thrombolytic activities of biogenic silver nanoparticles were evaluated by addition of nanoparticles to human blood samples under practical conditions. These green synthesized silver nanoparticles were found to have potent antibacterial, anticoagulant and thrombolytic properties which make them an attractive choice for future medical applications.

Keywords: biogenic silver nanoparticles; Euphorbia acruensis latex; UV-Vis spectroscopy; HRTEM; anticoagulant property; antibacterial activity

\section{Introduction}

Research in the field of green synthesis of nanoparticles continues to expand because of the abundance of biomaterials in nature, which are potent source of organic reductants for eco-benign production of nanoparticles. The green procedures avoid the use of harmful and expensive

\footnotetext{
* Corresponding author: Kaushik Roy, School of Materials Science and Nanotechnology, Jadavpur University, Kolkata-700032, India, e-mail: lordkaushikroy@gmail.com

Ambikesh K. Srivastwa and Chandan K. Ghosh, School of Materials Science and Nanotechnology, Jadavpur University, Kolkata-700032, India
}

chemicals for production of nanomaterials unlike chemical routes and the biogenic nanoparticles have shown considerable degree of biocompatibility with low toxicity [1,2]. In this context, several natural bio-products extracted from plants, micro-organisms and others have already been used for green production of nanomaterials [3-5]. Among different metallic nanoparticles developed so far, nanoparticles of silver have been the most anticipated one due to its diverse applications in the fields of optoelectronics, environmental remediation, electrochemistry and biomedicine as well [6-8]. Antibacterial and antifungal activities are the most investigated biological properties of silver nanoparticles till date $[9,10]$. But other biochemical properties of silver nanoparticles like anticoagulant and thrombolytic activities can also be explored to assess their potentiality in the field of haematology.

Euphorbia acruensis is a succulent plant (Figure 1) of Euphorbiaceae family and found in Africa, America and parts of South East Asia. It exudes milky white latex that contains active bio-reductants and capping agents like aldehydes, amines and aromatics [11]. This study focused on the use of E. acruensis latex for bio-reduction of silver ions in aqueous medium leading to the formation of silver nanoparticles. After green production, the nanoparticles were analysed using standard characterization tools like UV-Vis spectrometry, X-ray diffraction (XRD), transmission electron miscroscopy (TEM), Fourier transform infra-red spectroscopy (FTIR), etc. Finally, we demonstrated the antibacterial, anticoagulant and thrombolytic properties of these green synthesized silver nanoparticles following standard protocols [12]. This is a novel report on the use of Euphorbia acruensis latex for biosynthesis of silver nanoparticles along with the study of their diverse biological properties stated earlier.

\section{Materials and methods}

Euphorbia acruensis was collected from university garden (at Jadavpur University, India) and authenticated before the experiment. Analytical grade silver nitrate 
was purchased from Merck India Ltd. (Mumbai, India). Nutrient agar required for antibacterial assay was procured from Himedia (India). De-ionized (DI) water was required to perform all experimental work. The glasswares were cleaned with de-ionized water and dried prior to the experiments.

\subsection{Preparation of silver nanoparticles}

Euphorbia acruensis is a succulent plant that exudes milky white latex unlike cacti. At first, the Euphorbia acruensis was cleaned with DI water, chopped and the released milky-white latex was collected for experimental use. Silver nanoparticles were biologically prepared by addition of $50 \mathrm{~mL}$ plant extract to the equal amount of $20 \mathrm{mM}$ silver nitrate solution. The reacting mixture was observed at room temperature $\left(30^{\circ} \mathrm{C}\right)$ for next $12 \mathrm{~h}$. The reacting temperature was kept at $30^{\circ} \mathrm{C}$ to avoid any change in the composition of milky latex due to heating. After $15 \mathrm{~min}$ of addition, drastic change in the solution color (from colorless to light brown) was noticed indicating formation of metallic silver nanoparticles in the solution.

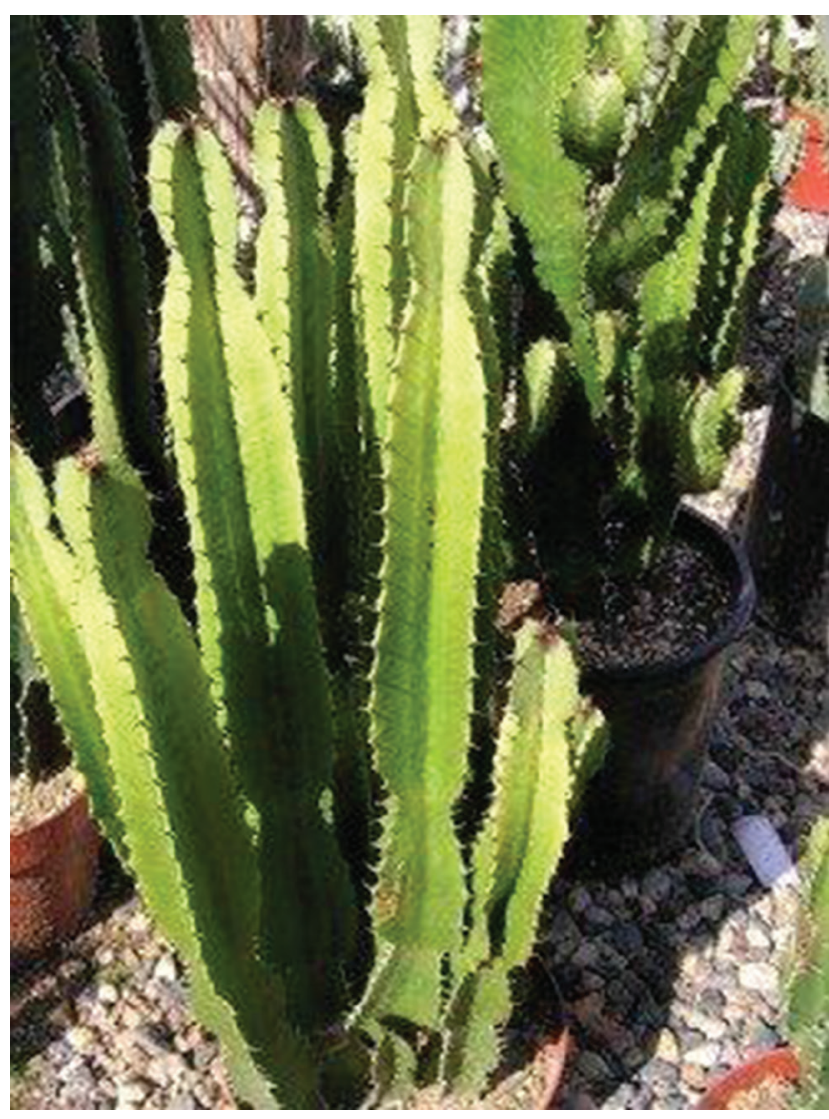

Figure 1: Euphorbia acruensis plant.

\subsection{Characterization of silver nanoparticles}

Generation of silver nanoparticles during reaction in the mixture was tracked at periodic intervals by scanning the mixture under double-beam UV-Vis spectroscope (Perkin Elmer, USA) and recording the UV-Vis spectra between 300 to $800 \mathrm{~nm}$ wavelengths. The produced nanoparticles were separated from the reacting solution by centrifuging it at $10,000 \mathrm{rpm}$ for $12 \mathrm{~min}$. The obtained soup after centrifugation was discarded and the pellet formed at the bottom (of centrifuge tube) was re-suspended in de-ionized water and centrifuged again to get rid of biomass residue completely. After repeated centrifugation, the precipitate was carefully collected and dried in a vacuum dryer overnight to obtain dry powder of silver nanoparticles. The XRD pattern of dry nanoparticles was recorded with the help of Rigaku Ultima-III X-ray diffractometer (CuKa radiation $\lambda=0.154 \mathrm{~nm}$; operating volt.- $40 \mathrm{kV} ; 2 \theta=20^{\circ}-80^{\circ}$ ). Fourier transform infra-red (FTIR) spectroscopy of these dry nanoparticles was performed on $\mathrm{KBr}$ pellet by using IR-Prestige FTIR spectroscope (Shimadzu, Japan) to identify the biomolecules associated with the stabilization of nanoparticles. Sample for high resolution TEM was carefully prepared by suspending dry silver nanoparticles in DI water keeping around $50 \mu \mathrm{g} / \mathrm{mL}$ concentration, i.e. a standard concentration for scanning nanoparticles under high resolution TEM. The suspension was further sonicated for $10 \mathrm{~min}$ and 2-3 drops of it were placed on carbon coated copper grid before drying it inside a desiccator. The grid was eventually scanned under high resolution TEM (Model name - JEOL-2010; operating volt.- $200 \mathrm{kV}$ ) to study the shape and morphology of biogenic silver nanoparticles. Energy dispersive X-ray (EDX) spectroscopy was performed by spreading 3-4 drops of this suspension on EDX sample holder and air-drying it prior to scanning under Inca X-stream EDX spectroscope (Oxford Instruments, UK).

\subsection{Antibacterial assay}

Antibacterial property of green synthesized silver nanoparticles was evaluated following agar disc diffusion method. Inoculates of two bacterial species- Pseudomonas putida and Staphylococcus aureus were prepared by growing single bacterial colony in the favourable nutrient broth medium overnight. The bacterial species were evenly spread on agar discs before creation of cups or wells on the discs. The suspension of biogenic silver nanoparticles 
prepared for TEM study (with concentration $50 \mu \mathrm{g} / \mathrm{mL}$ ) was used as sample A while the half-diluted part (i.e. concentration $25 \mu \mathrm{g} / \mathrm{mL}$ ) of it was taken as sample B for performing quantitative antibacterial test. Pure exudation of $E$. acruensis was taken as a negative control and labelled as sample $\mathrm{C}$ in this study. The three samples - A, B and C were added in three different wells created on each agar disc seeded with a specific bacterial species. These discs were then incubated overnight inside an incubator at $37^{\circ} \mathrm{C}$ and the zone of inhibition formed around wells after $24 \mathrm{~h}$ were measured to assess the antibacterial efficacy of these nanoparticles. The agar discs were maintained in triplicates for data acquisition of antibacterial assay with more accuracy.

\subsection{Study of anticoagulant and thrombolytic property}

The anticoagulant activity of the biogenic silver nanoparticles was evaluated on freshly collected human blood at room temperature. In this study, $1 \mathrm{~mL}$ suspension of silver nanoparticles (concentration $50 \mu \mathrm{g} / \mathrm{mL}$ ) was added to $10 \mathrm{~mL}$ fresh human blood (vial B). Another $10 \mathrm{~mL}$ of blood without addition was taken as a control (vial A).The two blood samples were then observed for next $1 \mathrm{~h}$ at room temperature for any noticeable changes.

Thrombolytic property of these nanoparticles was tested by dissolving fresh human blood clots in a clinical setup. Initially, 2-3 drops of fresh blood were spread on clean glass slide and allowed to form clot. $0.5 \mathrm{~mL}$ suspension of silver nanoparticles (concentration $50 \mu \mathrm{g} / \mathrm{mL}$ ) was then added to it. The blood clot sample was then observed visually as well as microscopically at room temperature for next $60 \mathrm{~min}$ to have an insight into various stages of thrombolysis.

\section{Results and discussion}

\subsection{Green synthesis of silver nanoparticles}

In order to utilize the reducing abilities of the functional organic molecules present in the latex, the milky latex of E. acruensis was incubated with silver nitrate solution as described above and the UV-Vis spectra at regular intervals were obtained [13]. As expected, silver nanoparticles were generated as the components of the exudation targeted silver ions present in the solution and reduced them to nanoscale metallic silver. The change of solution color from colorless to light brown after $15 \mathrm{~min}$ of incubation was a firm indication of the production of silver nanoparticles (Figure 2). The solution color intensified to dark brown and after $24 \mathrm{~h}$, no further change was visually observed indicating saturation of nanoparticle formation. The spectrometric data corroborated the observations as the absorbance reached its peak value at $420 \mathrm{~nm}$ which can be attributed to the wavelength of the surface plasmonic vibrations of metallic nano-silver (Figure 3) [14]. Figure 3 also demonstrates that the peak absorption value rose with incubation period and finally saturated after $24 \mathrm{~h}$ according to visual observance. The green synthesis was performed at different $\mathrm{pH}$ levels of the medium and no significant change was noticed for different $\mathrm{pH}$ values. This peak value of absorbance of the reacting medium increased with incubation time possibly due to production of more number of nanoparticles in the solution till arriving the state of equilibrium [15].

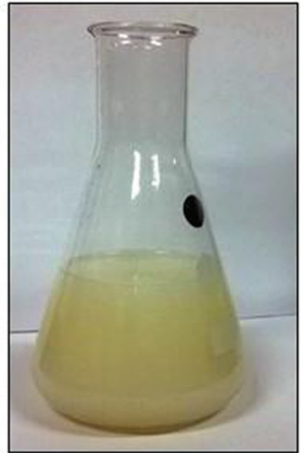

$0 \mathrm{~min}$

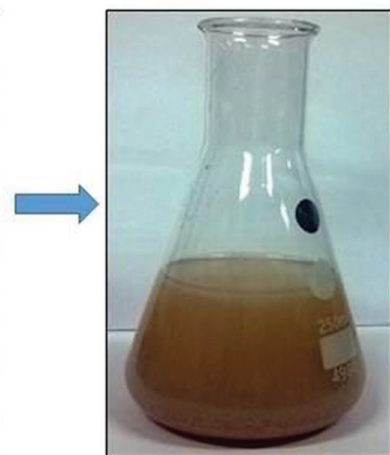

$15 \mathrm{~min}$

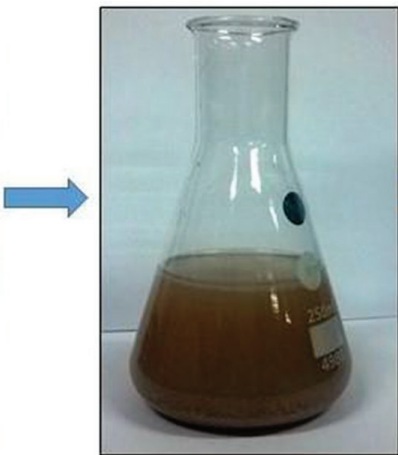

$60 \mathrm{~min}$

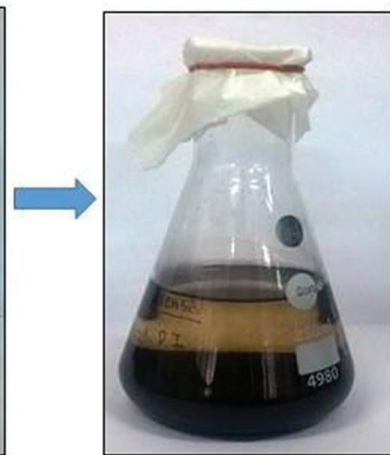

$24 \mathrm{~h}$

Figure 2: Colour change of reacting solution with time. 


\subsection{Characterization analysis}

X-ray diffraction pattern of dry nanoparticles was obtained to study the phase and crystallographic structures of the nanoparticles. The pattern consists of seven peaks (Figure 4) at $2 \theta$ values of $27.8^{\circ}, 32.2^{\circ}$, $46.25^{\circ}, 54.75^{\circ}, 57.4^{\circ}, 67.4^{\circ}$ and $76.76^{\circ}$ corresponding to (220), (122), (231), (331), (241), (104) and (311) planes

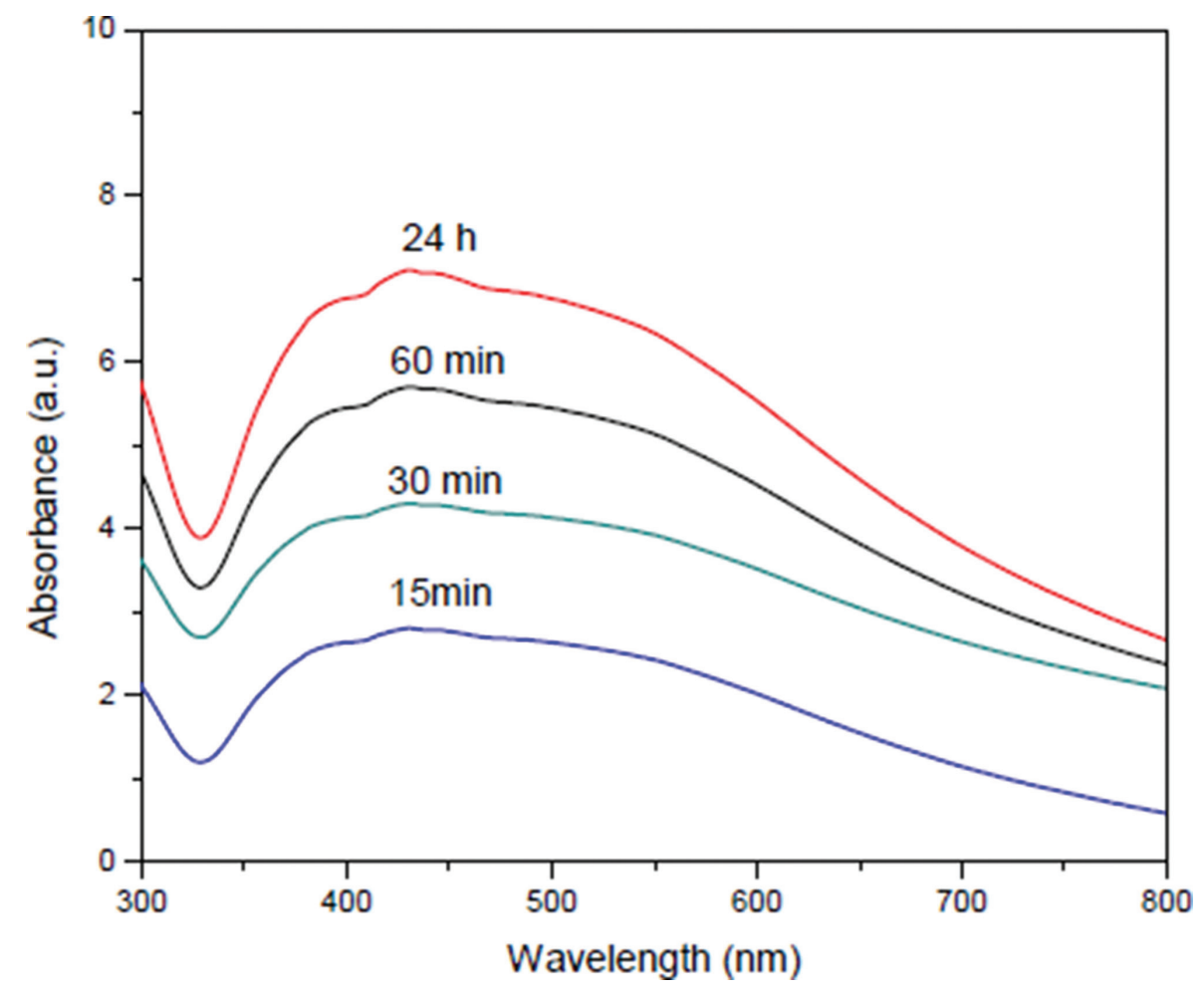

Figure 3: UV-Vis spectra of reacting medium at specific intervals.

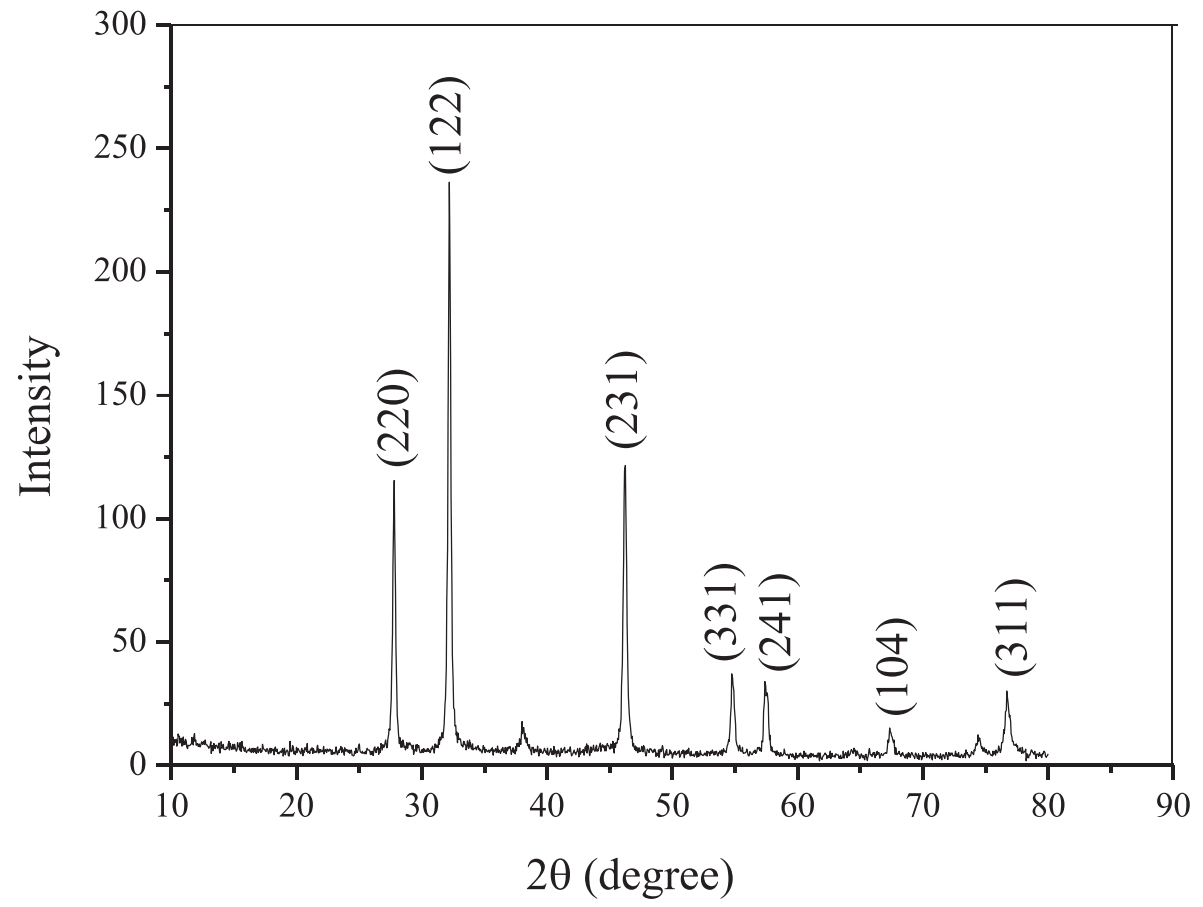

Figure 4: XRD pattern of silver nanoparticles. 
of silver respectively as observed and correlated to the powder diffraction card of JCPDS (file no. 4-783) [16]. These findings confirm that the nanoparticles have face centred cubic (fcc) crystal structure. The crystalline size was estimated following Debye-Scherrer's equation and it was found to be around $35 \mathrm{~nm}$.

Figure 5 shows the TEM images of green synthesized silver nanoparticles where the particles were observed to be closely spherical-shaped. The size distribution profile was obtained by counting at least 200 nanoparticles and the histogram is given in Figure 5d. From histogram, it is evident that most of the particles belong to the size range of 10 to $40 \mathrm{~nm}$ which is in accordance with the result of XRD. From Figure $5 c$, the interplanar spacings were manipulated to be 0.27 and $0.32 \mathrm{~nm}$ that may correspond to (122) and (220) crystal planes of silver nanoparticles respectively.
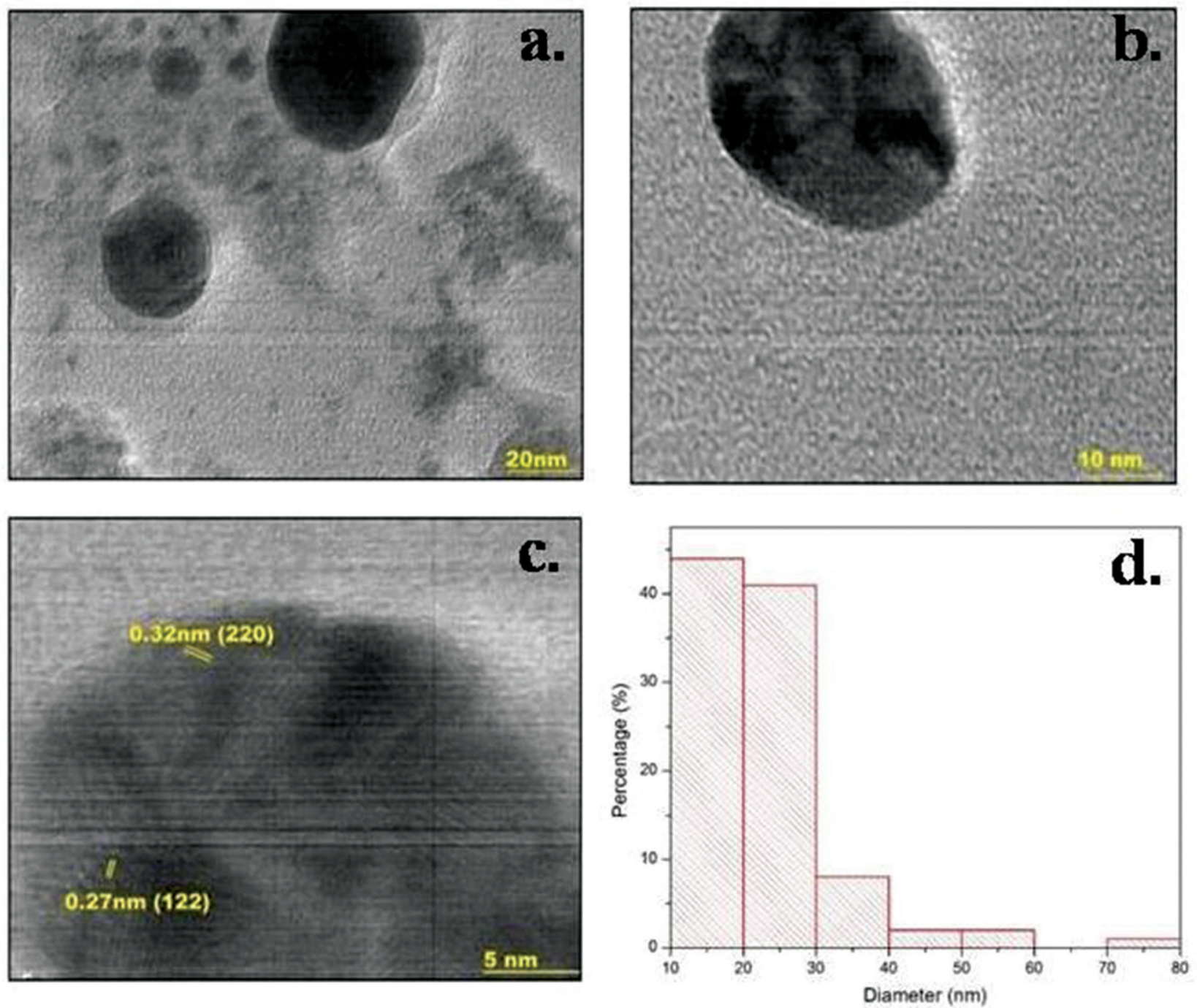

FTIR spectroscopy was performed to have an insight into the capping and stabilization mechanism of nanoparticles during interaction with Euphorbia acruensis latex. The recorded FTIR spectra of E. acruensis latex and biogenic silver nanoparticles are shown in Figure 6. The spectrum of plant latex consists of eight noticeable peaks which can be correlated to the standard IR database to identify the organic molecules responsible for reduction and capping. The peaks at 1649 (peak 6) and $3397 \mathrm{~cm}^{-1}$ (peak 8) indicate stretching of $\mathrm{C}=\mathrm{O}$ and $\mathrm{O}-\mathrm{H}$ bonds present in amides and alcohols respectively [17]. A noticeable band present at $1436 \mathrm{~cm}^{-1}$ (peak 5) may be attributed to stretching of benzene ring present in aromatic compounds like ascorbic acid, phenol etc. [18]. Two vibrational bands at 2941 (peak 7) and $887 \mathrm{~cm}^{-1}$ (peak 3) may correspond to stretching and bending of $\mathrm{C}-\mathrm{H}$ bonds present in alkanes and alkenes

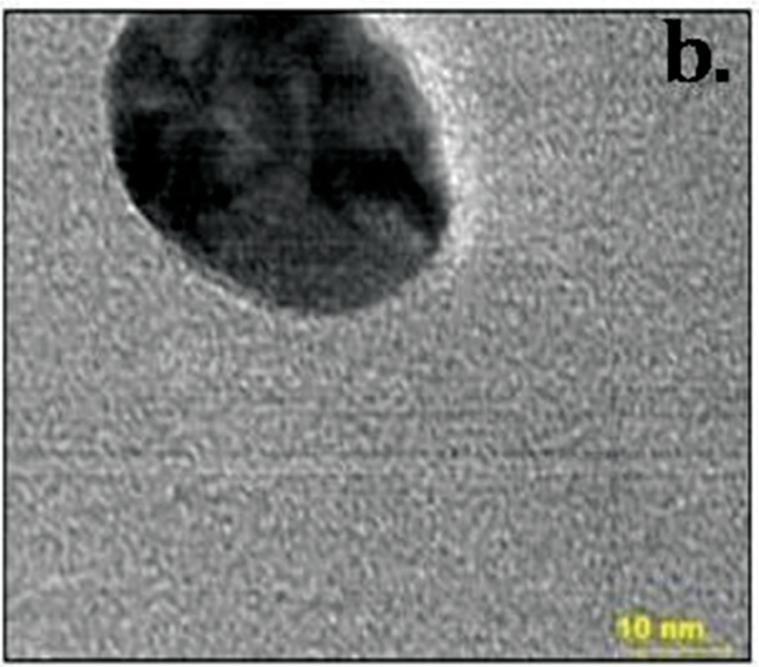

Figure 5: HRTEM images and histogram of silver nanoparticles. 


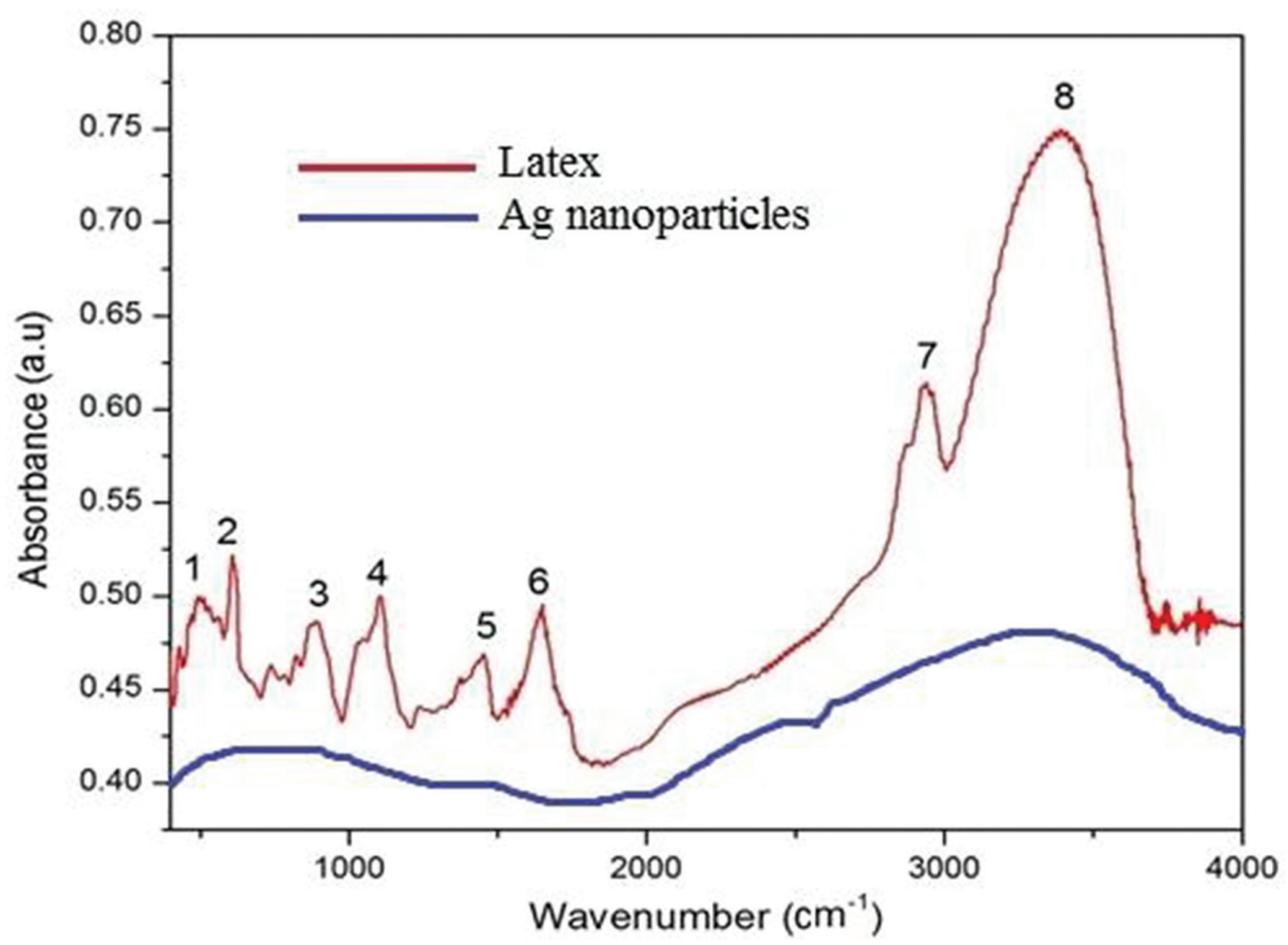

Figure 6: FTIR spectra of plant latex and biogenic silver nanoparticles.

respectively [19]. The rest of the peaks (peak 1, 2 and 4) denote the stretching of carbon-halogen bonds present in alkyl halides. On the other hand, the spectrum of green synthesized silver nanoparticles features no significant absorbance peak indicating thorough removal of biomass residue from particle surface after production and eventually high level of purity of the biogenic nanoparticles.

The presence of aromatic compounds (like ascorbic acid, phenol etc.) along with amides and aldehydes in the plant latex probably indicates that these organic molecules played the key role of capping and stabilizing nanoparticles during interaction of plant exudation with metal ions in the reacting solution $[19,20]$.

EDX spectroscopy was employed to analyse the purity and weight percentage of metallic silver in the produced nanoparticles. The result of EDX (Figure 7) shows a couple of silver peaks and the weight percentage of Ag was found to be $74 \%$. This data further confirms the production and purity of metallic silver. Small peaks of carbon, oxygen and chlorine were observed probably due to the presence of organic capping agents on the surface of particles [21].

\subsection{Study of antibacterial property}

Antibacterial property of green synthesized silver nanoparticles was tested towards a couple of pathogenic bacteria - P. putida and S. aureus. Pseudomonas putida is a gram-negative bacteria whereas Staphylococcus aureus is a gram-positive type. As seen from Figure 8, the larger inhibition zone was noticed against $P$. putida irrespective of the concentration of the nanoparticles added. S. aureus showed comparatively lower inhibition levels. Quantitative analysis of inhibitory effect against the tested bacterial species indicated a sharp rise in the inhibitory effect depending on higher levels of concentrations of biogenic nanoparticles (Table 1). The plant latexexhibited zero inhibition denoting no involvement in the antibacterial process. The better antibacterial activity of silver nanoparticles towards gram-negative $P$. putida may be due to the difference in cell wall compositions of grampositive and gram-negative bacteria [22]. Cell wall of gram positive bacteria is composed of multiple thicker layers of bio-polymer called peptidoglycan whereas the cell wall of gram negative bacteria (like P. putida) consists of only single or double layers of it [23]. Therefore, the penetration 


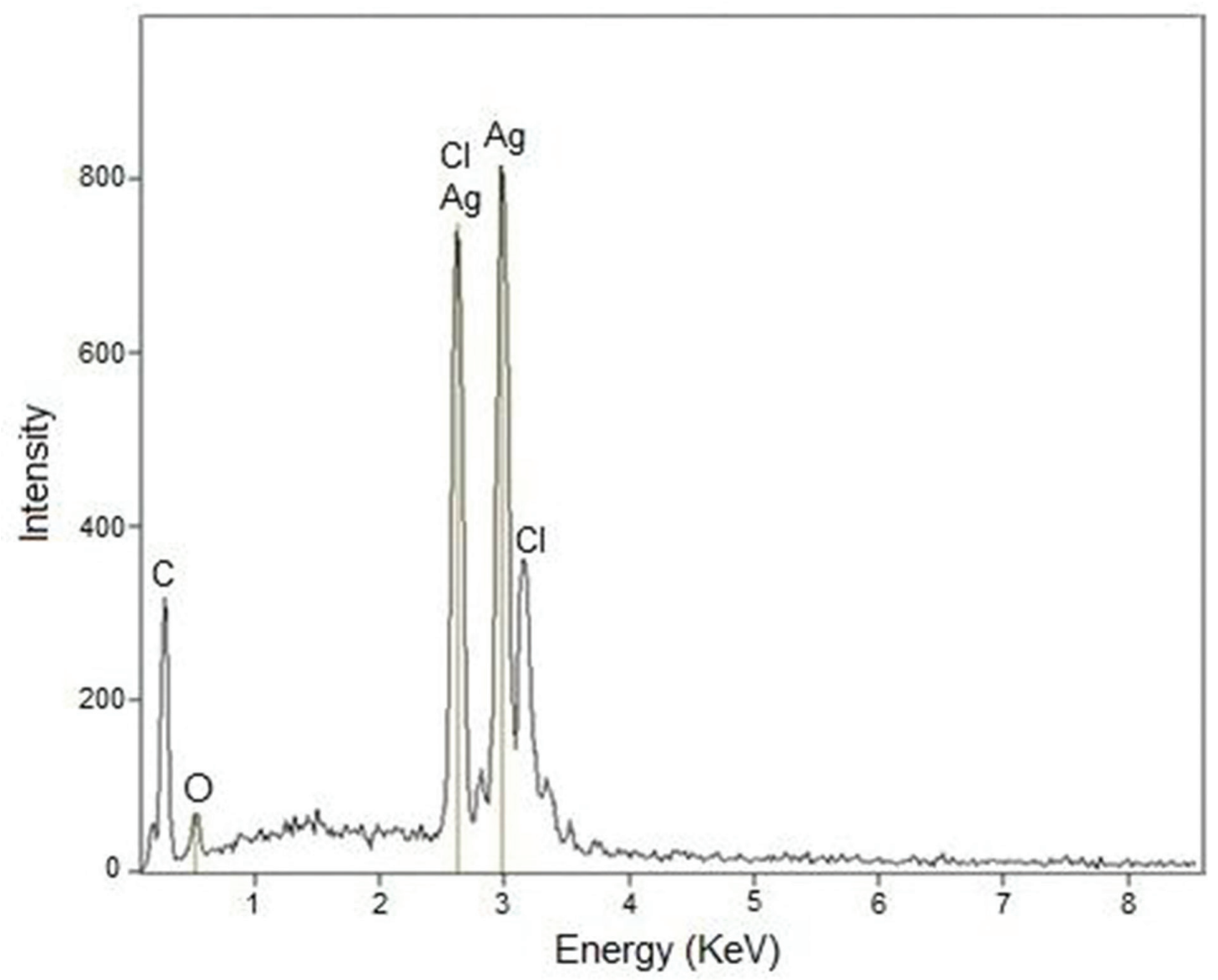

Figure 7: EDX spectrum of green synthesized nanoparticles.
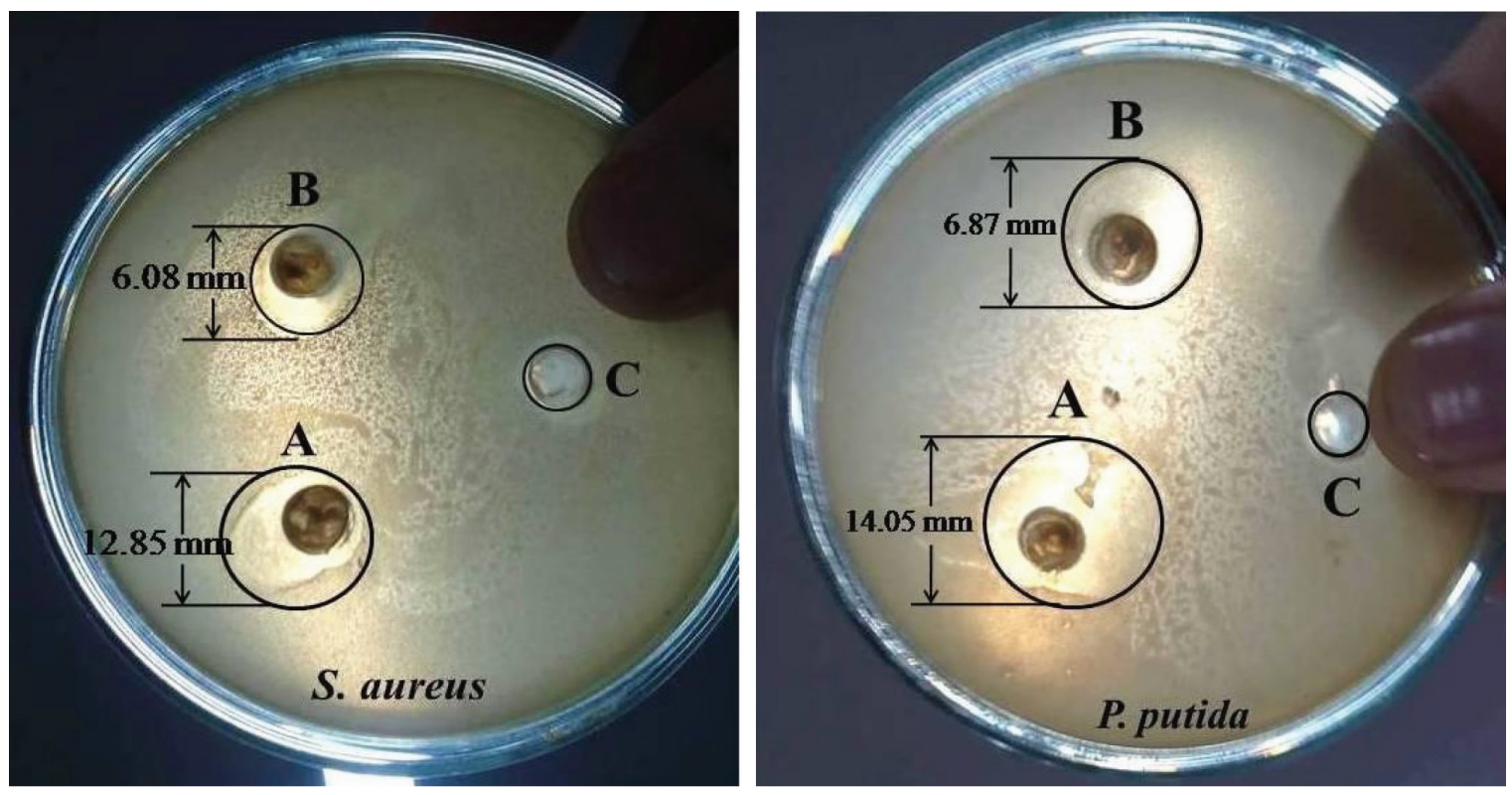

Figure 8: Result of antibacterial disc diffusion assay. 
of colloidal particles into the cellular system was easier in case of gram negative bacteria than gram positive.

Although the entire antibacterial mechanism of silver nanoparticles is not elucidated yet, but a few proposed theories are available in the literature to comprehend the action of metal nanoparticles on microbial cells. A few reports claim that the gold or silver nanoparticles have the ability to be attached on the bacterial cell wall due to electrostatic attraction and then penetrate it forming holes or 'pits' [24]. The formation of 'pits' on cell membrane alters the membrane permeability causing leakage of cellular fluid. Due to leakage of fluid, cellular transport degrades and the cells fail to survive for too long [25]. Another possible factor for cell death may be the metal ions which are released from nanoparticles during encounter with bacterial cells. The metal ions inhibit respiratory enzymes and break the cellular respiratory chain resulting into reactive oxygen species (ROS) generation $[9,26]$. ROS can impart oxidative stress to bacterial cells and eventually the cells cease all regular functions and expire [27].

\subsection{Anticoagulant and thrombolytic property}

Anticoagulant and thrombolytic activity of green synthesized nanoparticles was tested by addition of

Table 1: Result of antibacterial assay.

\begin{tabular}{lrr}
\hline $\begin{array}{l}\text { Tested bacterial } \\
\text { species }\end{array}$ & $\begin{array}{r}\text { Concentration of silver } \\
\text { nanoparticles in } \\
\text { suspension }(\mu \mathrm{g} / \mathrm{mL})\end{array}$ & $\begin{array}{r}\text { Inhibition zone } \\
\text { diameter }(\text { mean of } \\
\text { triplicates) }(\mathrm{mm})\end{array}$ \\
\hline $\begin{array}{l}\text { Pseudomonas } \\
\text { putida }\end{array}$ & 0 (control) & - \\
& 25 & $6.87 \mathrm{~mm}$ \\
Staphylococcus & 50 & $14.05 \mathrm{~mm}$ \\
aureus & 0 (control) & - \\
& 25 & $6.08 \mathrm{~mm}$ \\
& 50 & $12.85 \mathrm{~mm}$ \\
\hline
\end{tabular}

nanoparticles to human blood samples and further observation. The control in vial A began to coagulate within 10 min of incubation. The blood sample thickened over time and finally formed thick blood clot after 60 min (Figure 9). On the other hand, the blood sample with addition of nanoparticles (in vial B) underwent no significant changes and eventually no mark of coagulation was observed after $60 \mathrm{~min}$ of incubation (Figure 9).

During the assessment of thrombolytic property, the suspension of biogenic nanoparticles was added to a preformed blood clot for further observation. Immediately after addition, the blood clot on the glass slide began to be liquefied gradually and was dissolved completely after 30 min as shown in Figure 10.

The mechanisms for anticoagulant and thrombolytic activity of silver nanostructures are not theoretically decoded yet, but probable biochemical mechanism can be explained in the light of thrombolysis process. Biogenic silver nanoparticles may involve into the inhibition of enzymes which are responsible for generating blood clotting proteins [28]. Probably nano-silver inhibits the conversion of prothrombin into thrombin which is the key factor for producing insoluble strands of fibrin and catalysing other coagulating factors [29]. In addition, silver nanoparticles may be involved in activating enzymes that produce plasmin which can break cross-links of fibrin molecules and dissolve blood clots [30].

The result obtained here is in accordance with the thrombolytic activity of biochemical mediated silver nanoparticles reported by Harish et al. [31]. The images clearly indicated full dispersion of blood clot by green synthesized silver nanoparticles. While blood coagulation is an essential process to prevent excessive bleeding, timely dissolution of clots is equally necessary to curb thrombosis [32]. Conventional antithrombic treatments like streptokinase have limited scope of application due to short half-life, foreign agent neutralization and
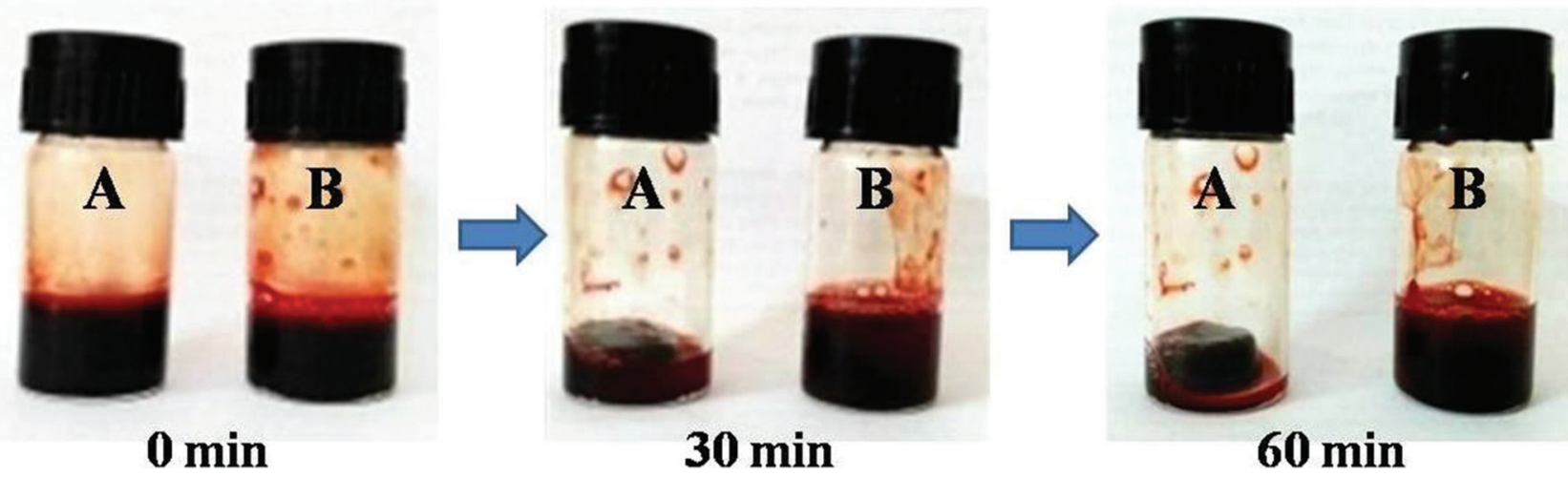

$30 \mathrm{~min}$

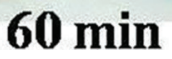

Figure 9: Anticoagulant activity of silver nanoparticles. 


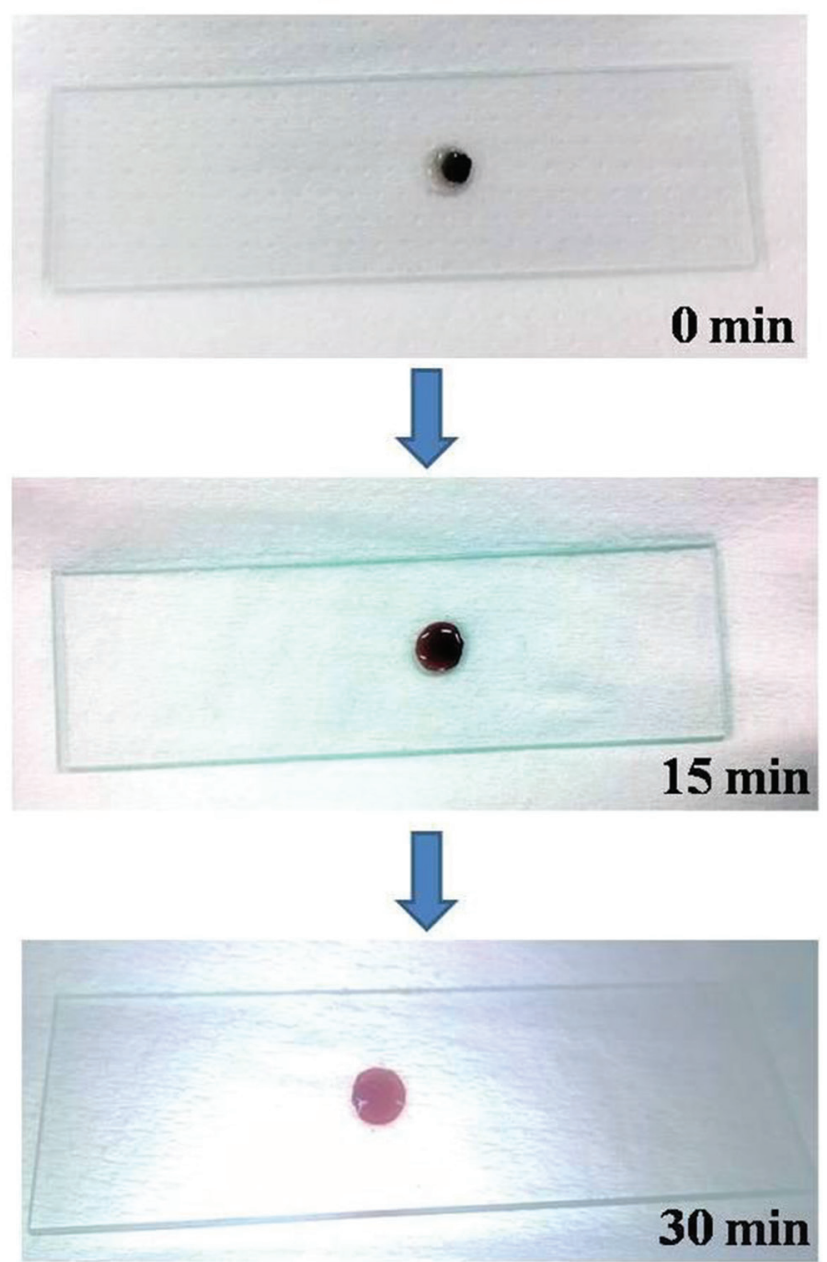

Figure 10: Thrombolytic activity of silver nanoparticles.

possibility of excessive bleeding [33]. Although very limited information is available in the literature on using nano-silver as thrombolytic agent, this report claims to have proved the potency of silver nanoparticles as anticoagulant and thrombolytic agent in the management of thrombosis. The potentiality shown by the biogenic silver nanoparticles in this study may have some useful applications in clinical domain for prevention of thrombosis and other disorders associated with it.

\section{Conclusion}

Plant extract-mediated green synthetic protocols of nanoparticles have drawn the attention of researchers for their fascinating, low cost and eco-benign features. In this report, we introduced a novel green platform where the milky white exudation of the plant- $E$. acruensis effectively reduced silver salt and produced silver nanoparticles in aqueous medium. Standard characterization tools were used to reveal the crystal phases and microstructure of the produced nanoparticles also to detect the role of biomolecules as stabilizing agents during interaction. The biosynthesized silver nanoparticles were proved to have obtrusive antibacterial efficacy towards gram positive and as well as gram negative bacterial strains. Most importantly, these nanoparticles were found to possess prominent anticoagulant and thrombolytic properties. These results extend the knowledge of bio-applications of silver nanoparticles and open up new possibilities in the domain of biomedical research.

\section{References}

[1] Irimia-Vladu M., “Green" electronics: biodegradable and biocompatible materials and devices for sustainable future. Chem. Soc. Rev., 2014, 43, 588-610.

[2] Saif S., Tahir A., Chen Y., Green synthesis of iron nanoparticles and their environmental applications and implications. Nanomater., 2016, 6(11), 209.

[3] Ashraf J.M., Ansari M.A., Khan H.M., Alzohairy M.A., Choi I., Green synthesis of silver nanoparticles and characterization of their inhibitory effects on AGEs formation using biophysical techniques. Sci. Rep., 2016, 6, 20414.

[4] Roy K., Ghosh C.K., Sarkar C.K., Selective amino acid detection by green synthesized copper nanoparticles prepared using basil (Ocimum tenuiflorum) flower extract. Microsyst. Technol., 2018, DOI:10.1007/s00542-018-4205-7.

[5] Ahmed S., Ahmad S.M., Swami B.L., Ikram S., Green synthesis of silver nanoparticles using Azadirachta indica aqueous leaf extract. J. Radiat. Res. 2016, 9(1), 1-7.

[6] Ismail R.A., Almashhadani N.J., Sadik R.H., Preparation and properties of polystyrene incorporated with gold and silver nanoparticles for optoelectronic applications. Appl. Nanosci., 2017, 7(3), 109-116.

[7] Roy K., Ghosh C.K., Measurement of electrical conductivity of thin film composed of green synthesized copper nanoparticles. $1^{\text {st }}$ Intern. Conf. Emerg. Trend. Electron. Dev. Comput. Tech. (EDCT), 2018, DOI:10.1109/EDCT.2018.8405073.

[8] Giner-Casares J.J., Henriksen-Lacey M., Coronado-Puchau M., Liz-Marzan L.M., Plasmonic surfaces for cell growth and retrieval triggered by near-infrared light. Mater. Today, 2016, 19(1), 19-28.

[9] Prabhu S., Poulose E.K., Silver nanoparticles: mechanism of antimicrobial action, synthesis, medical applications, and toxicity effects. Int. Nano Lett., 2012, 2(32), 1-10.

[10] Roy K., Ghosh C.K., Biological synthesis of metallic nanoparticles: A green alternative (Chapter 7), Nanotechnology: Synthesis to Applications. CRC Press, 2017, ISBN: 978-1-138-03273-6, 153-168. 
[11] Spano D., Pintus F., Mascia C., Scorciapino M.A., Casu M., Floris G., et al., Extraction and characterization of a natural rubber from Euphorbia characias latex. Biopolymers, 2012, 97(8), 589-594.

[12] Ghatage S.L., Navale S.S., Mujawar N.K., Patil S., Patil V., Antimicrobial screening. Ind. J. Drug., 2014, 2(3), 84-88.

[13] Ioannidis A.S., Papageorgiou K.I., Andreou P.S., Exposure to Euphorbia lathyris latex resulting in alkaline chemical injury: a case report. J. Med. Case Rep., 2009, 3, 115.

[14] Roy K., Ghosh C.K., Sarkar C.K., Rapid colorimetric detection of $\mathrm{Hg}^{2+}$ ion by green silver nanoparticles synthesized using Dahlia pinnata leaf extract. Green Process. Synth., 2015, 4(6) 455-461.

[15] Sasikala D., Govindaraju K., Tamilselvan S., Singaravelu G., Soybean protein: A natural source for the production of green silver nanoparticles. Biotechnol. Bioprocess. Eng., 2012, 17(6), 1176-1181.

[16] Li H.J., Zhang A.Q., Hu Y., Sui L., Qian D.J., Chen M., Large-scale synthesis and self-organization of silver nanoparticles with Tween 80 as a reductant and stabilizer. Nanoscale Res. Lett., 2012, 7, 612.

[17] Umer A., Naveed S., Ramzan N., Rafique M.S., Imran M., A green method for the synthesis of Copper Nanoparticles using L-ascorbic acid. Matéria (Rio J.), 2014, 19(3), 197-203.

[18] Ahmad S.I., Syed I.A., Prasad P.R., Ahmad A., Quantitation of urea in urine by Fourier transforms infrared spectroscopy, Der Pharma Chemica, 2014, 6(1), 90-96.

[19] Ahmmad S.K., Samee M.A., Taqiullah S.M., Rahman S., FT-IR and Raman spectroscopic studies of $\mathrm{ZnF}_{2}-\mathrm{ZnO}-\mathrm{As}_{2} \mathrm{O}_{3}-\mathrm{TeO}_{2}$ glasses. J. Taibah Univ. Sci., 2016, 10(3), 329-339.

[20] Shetgiri N.P., Nayak B.K., Synthesis and antimicrobial activity of some succinimides. Ind. J. Chem., 2005, 44B, 1933-1936.

[21] Anandalakhsmi K., Venugobal J., Ramasamy V., Characterization of silver nanoparticles by green synthesis method using Pedalium murex leaf extract and their antibacterial activity. Appl. Nanosci., 2016, 6(3), 399-408.

[22] Kruk T., Szczepanowicz K., Stefanska J., Socha R.P., Warszynski P., Synthesis and antimicrobial activity of monodisperse copper nanoparticles. Colloid. Surface B, 2015, 128, 17-22.
[23] Aziz N., Faraz M., Pandey R., Shakir M., Fatma T., Varma A., et al., Facile algae-derived route to biogenic silver nanoparticles: synthesis, antibacterial, and photocatalytic properties. Langmuir, 2015, 31, 11605-11612.

[24] Shi T., Sun X., He Q., Cytotoxicity of silver nanoparticles against bacteria and tumor cells. Curr. Protein Pept. Sci., 2018, 19(6), 525-536.

[25] Roy K., Ghosh C.K., Environmental and biological applications of nanoparticles (Chapter 8), Nanotechnology: Synthesis to Applications. CRC Press, 2017, ISBN: 978-1-138-03273-6, 169-192.

[26] Chakrapani V., Ahmed K.B.A., Kumar V.V., Ganapathy V., Anthony S.P., Anbazhagan V., A facile route to synthesize casein capped copper nanoparticles: an effective antibacterial agent and selective colorimetric sensor for mercury and tryptophan. RSC Adv., 2014, 4, 33215-33221.

[27] Dobrucka R., Dlugaszewska J., Biosynthesis and antibacterial activity of $\mathrm{ZnO}$ nanoparticles using Trifolium pratense flower extract. Saudi J. Biol. Sci., 2016, 23(4), 517-523.

[28] Huang H., Lai W., Cui M., Liang L., Lin Y., Fang Q., et al., An evaluation of blood compatibility of silver nanoparticles. Sci. Rep., 2016, 6, 25518.

[29] Lateef A., Akande M.A., Ojo S.A., Folarin B.I., Gueguim-Kana E.B., Beukes L.S., Paper wasp nest-mediated biosynthesis of silver nanoparticles for antimicrobial, catalytic, anticoagulant, and thrombolytic applications. 3 Biotech., 2016, 6, 140.

[30] Cheng R., Huang W., Huang L., Yang B., Mao L., Jin K., et al., Acceleration of tissue plasminogen activator-mediated thrombolysis by magnetically powered nanomotors. ACS Nano, 2014, 8(8), 7746-7754.

[31] Harish B.S., Uppuluri K.B., Anbazhagan V., Synthesis of fibrinolytic active silver nanoparticle using wheat bran xylan as a reducing and stabilizing agent. Carbohyd. Polym., 2015, 132, 104-110.

[32] Chapin J.C., Hajjar K.A., Fibrinolysis and the control of blood coagulation. Blood Rev., 2015, 29(1), 17-24.

[33] Banerjee A., Chisti Y., Banerjee U.C., Streptokinase--a clinically useful thrombolytic agent. Biotech. Adv., 2004, 22, 287-307. 